\title{
A Brucella Omp16 Conditional Deletion Strain Is Attenuated in BALB/c Mice
}

\author{
Feijie Zhi ${ }^{1}$, Jiaoyang Fang ${ }^{1}$, Weifang Zheng ${ }^{1}$, Junmei Li ${ }^{1}$, Guangdong Zhang ${ }^{1}$, Dong Zhou ${ }^{1,2}$, \\ Yaping Jin $^{1,2}$, and Aihua Wang ${ }^{1,2 *}$ \\ ${ }^{1}$ College of Veterinary Medicine, Northwest A\&F University, Yangling 712100, P.R. China \\ ${ }^{2}$ Key Laboratory of Animal Biotechnology of the Ministry of Agriculture, Northwest A\&F University, Yangling \\ 712100, P.R. China
}

\begin{abstract}
Brucella spp. are facultative intracellular pathogens that invade, survive and proliferate in numerous phagocytic and non-phagocytic cell types, thereby leading to human and animal brucellosis. Outer membrane proteins (Omps) are major immunogenic and protective antigens that are implicated in Brucella virulence. A strain deleted of the omp16 gene has not been obtained which suggests that the Omp16 protein is vital for Brucella survival. Nevertheless, we previously constructed an omp 16 conditional deletion strain of Brucella, $\Delta O \mathrm{mp} 16$. Here, the virulence and immune response elicted by this strain were assessed in a mouse model of infection. Splenomegaly was significantly reduced at two weeks post-infection in $\Delta 0 \mathrm{mp} 16$-infected mice compared to infection with the parental strain. The bacterial load in the spleen also was significantly decreased at this post-infection time point in $\Delta$ Omp16-infected mice. Histopathological changes in the spleen were observed via hematoxylineosin staining and microscopic examination which showed that infection with the $\Delta 0 \mathrm{mp} 16$ strain alleviated spleen histopathological alterations compared to mice infected with the parental strain. Moreover, the levels of humoral and cellular immunity were similar in both $\Delta$ Omp 16 -infected mice and parental strain-infected mice. The results overall show that the virulence of $\Delta 0 \mathrm{mp} 16$ is attenuated markedly, but that the immune responses mediated by the deletion and parental strains in mice are indistinguishable. The data provide important insights that illuminate the pathogenic strategies adopted by Brucella.
\end{abstract}

Keywords: Brucella suis S2, Omp16, virulence, immune response, mice

Received: July 7, 2021 Accepted: October 18, 2021

First published online: October 20, 2021

*Corresponding author Phone: +862987082869 E-mail: aihuawang1966@ 163.com

pISSN 1017-7825 elSSN 1738-8872

Copyright $@ 2022$ by the authors. Licensee KMB. This article is an open access article distributed under the terms and conditions of the Creative Commons Attribution (CC BY) license

\section{Introduction}

Brucellosis is an endemic zoonotic bacterial disease caused by Brucella spp. which are able to establish persistent infection in hosts $[1,2]$. The Brucella genus currently comprises six classical species based on natural host specificity [3], although novel Brucella species continue to be discovered, including Brucella ceti, B. inopinata, $B$. microti, and B. pinnipedialis [4, 5]. Among the most well-characterized species, B. abortus, B. canis, B. melitensis, and $B$. suis infect humans with most cases attributed to B. melitensis [6, 7]. The main symptoms of Brucella infection in non-human animals are spontaneous abortion at late gestation, infertility and orchitis which lead to devastating economic and social losses [8-10]. In contrast, brucellosis in humans is characterized by a long incubation period that leads to a variety of atypical clinical symptoms, including undulant fever, osteoarticular pain, splenomegaly, and hepatomegaly. More than 500,000 human cases of Brucella infection are reported annually worldwide [7]. Brucella vaccines that currently are adminstered in animals have certain disadvantages, including sero-diagnostic interference and residual pathogenicity [11-13]. No approved Brucella vaccines are available for human use [9].

One of the main characteristics of Brucella spp. is the absence of certain classic virulence factors, including cytolysins, fimbria, exotoxins, capsules, lysogenic phages, and plasmids, compared to other pathogenic bacteria [14]. Instead, the main virulence factors in Brucella include type IV secretion systems (T4SS), lipopolysaccharides (LPS), the BvrR/BvrS two-component system, cyclic $\beta$-1-2-glucans (C $\beta G$ ), and outer membrane proteins (Omps) that mediate interaction with the host cell surface or related signaling pathways to promote bacterial survival [7, 15, 16]. A functional T4SS system is required for Brucella survival in vivo and in vitro, and T4SS effectors, such as VceC, BspA, BspB, and BspB, contribute to Brucella intracellular proliferation [17, 18]. LPS in Brucella exhibits properties that differ from LPS in other gram-negative bacteria. For example, LPS of Brucella is less toxic and less active than Escherichia coli LPS [16]. Brucella $\mathrm{C} \beta \mathrm{G}$ is required for survival in HeLa cells and proliferation in mice [19]. In addition, other factors involved in Brucella virulence and that play different roles in pathogenicity include urease, cytochrome oxidase, nitric oxide reductase, and Brucella virulence factor A (BvfA) $[15,16]$.

Omps in Brucella are exposed at the bacterial surface and are implicated strongly in virulence [20, 21]. For 
example, the Omp10 deletion strain is defective for growth in minimal medium and infection is attenuated in mice $[22,23]$. Deletion of Omp19 reduces intracellular survival in macrophages and also attenuates murine infection $[24,25]$. Omp 25 and Omp31 also contribute to intracellular survival of Brucella in vitro and in vivo, as well as to chronic infection $[20,26]$. Omp 16 is a pathogen-associated molecular pattern (PAMP) that is highly conserved in all Brucella species and which activates dendritic cells (DCs) and which also induces a T helper 1 (Th1) immune response [27]. In addition, Omp16 can alter Brucella-mediated immune-related pathways in macrophages. However, Brucella deleted of the omp16 gene has not been acquired, which suggests that the Omp16 protein is vital for survival in this bacterium [28]. Nevertheless, we previously generated a derivative of B. suis strain 2 (B. suis S2) in which omp16 expression was controlled ( $\Delta \mathrm{Omp} 16)$. However, the virulence and immune response mediated by the $\Delta$ Omp16 strain have not been evaluated in mice.

In this study, our goal was to examine the effect of Omp16 on the virulence and immunity of Brucella in BALB/ c mice by analyzing splenomegaly, spleen bacterial load, spleen histopathology, and humoral and cellular immunity. We show that Omp16 is required for Brucella survival in vivo and that the levels of humoral and cellular immunity in $\Delta$ Omp16-infected mice were similar to those in wild-type strain-infected mice. These findings indicate that Omp16 is important for the virulence of Brucella, but that the immune response is not altered by the absence of the protein. The study provides new insights into the crucial role of Omp16 in Brucella virulence and into a broader understanding of the mechanisms of Brucella pathogenicity.

\section{Materials and Methods}

\section{Bacterial Strains and Culture Conditions}

Wild-type B. suis S2 (CVCC reference number CVCC70502) and $\triangle$ Omp16 have been described previously [29]. B. suis S2 and $\Delta$ Omp16 were grown on tryptic soy agar (TSA; Sigma, USA) for $72 \mathrm{~h}$ at $37^{\circ} \mathrm{C}$ and $5 \% \mathrm{CO}_{2}$ and then cultured in tryptic soy broth (TSB; Sigma) at $37^{\circ} \mathrm{C}$ with shaking to an optical density at $600 \mathrm{~nm}\left(\mathrm{OD}_{600}\right)$ of $\sim 0.6$. For infection, B. suis $\mathrm{S} 2$ and $\Delta \mathrm{Omp} 16$ in the logarithmic growth phase were collected by centrifugation and the numbers of bacteria were determined using 10 -fold gradient dilutions.

\section{Mice Infection}

Groups of six-to-eight-week old BALB/c female mice (Experimental Animal Center, Xi'an Jiaotong University, Shaanxi, China) were acclimated at least one week prior to infection. Each experimental group contained at least five mice. The mice were inoculated intraperitoneally with $10^{7}$ colony-forming units (CFU) of B. suis S2 or with the $\Delta$ Omp16 strain in $200 \mu \mathrm{l}$ of phosphate-buffered saline (PBS). The infected mice were monitored daily for survival. The mice were sacrificed by cervical dislocation at one, two and four weeks post-infection. The spleens from infected mice were removed and weighed to evaluate splenomegaly. To determine the bacterial load, onethird of each spleen was homogenized in $0.5 \mathrm{ml}$ of PBS. Tissue homogenates were diluted by a 10 -fold gradient and spread on TSA plates. CFU were counted after $72 \mathrm{~h}$ of incubation at $37^{\circ} \mathrm{C}$.

\section{Histological and Immunohistological Analysis}

Spleen tissues from infected mice were collected at one, two and four weeks post-infection. One-third of each spleen was fixed in $4 \%$ paraformaldehyde for three days at room temperature. After fixation, the tissues were embedded in paraffin blocks which were then sectioned into $5 \mu \mathrm{m}$ slices using a microtome. The sections were transferred to glass slides and allowed to adhere prior to further processing. The sections were examined routinely with hematoxylin-eosin staining (H\&E staining) to evaluate pathological features. Immunohistochemical staining was performed to observe the bacterial load in spleen samples. Then, the sections were then treated with $3 \% \mathrm{H}_{2} \mathrm{O}_{2}$ for $30 \mathrm{~min}$ at room temperature to block endogenous peroxidase activity. Next, the sections were blocked in PBS with $1 \%$ BSA for $1 \mathrm{~h}$ at room temperature before being permeabilized in PBS containing $0.25 \%$ Triton X100 at room temperature for $30 \mathrm{~min}$. Finally, the sections were incubated with primary anti-brucella goat polyclonal antibody (1:100 dilution) overnight at $4^{\circ} \mathrm{C}$. Donkey anti-goat Alexa Fluor 555 (Invitrogen, USA; 1:500 dilution) was used as the secondary antibody. Subsequently, slides were observed under a laser scanning confocal microscope (Nikon).

\section{RNA Isolation and Quantitative Real-Time PCR}

Spleen tissues from infected mice were collected at one, two and four weeks post-infection. One-third of each spleen was homogenized in $0.5 \mathrm{ml}$ of TRIzol (Invitrogen). Total RNA was extracted from the spleen tissues using TRIzol according to the manufacturer's protocol. Reverse transcription was performed using the RevertAid First Strand cDNA Synthesis Kit (Thermo Fisher Scientific, USA) according to the manufacturer's recommended protocols. SYBR Premix Ex Taq (Vazyme, China) and the ABI 7500 Sequencing Detection System (Applied Biosystems, USA) were used for quantitative real-time PCR (qRT-PCR). The relative transcription levels were analyzed by the $2^{-\Delta \Delta \mathrm{Ct}}$ method [30]. All the primers used are listed in Table 1 .

Table 1. A list of all primers used in qRT-PCR.

\begin{tabular}{cccc}
\hline Gene & Forward primer (5'-3') & Reverse primer (5'-3') & Length (bp) \\
\hline IFN- $\gamma$ & AGCAACAACATAAGCGTCA & GTGGACCACTCGGATGAG & 156 \\
TNF- $\alpha$ & TCTCATTCCTGCTTGTGGC & CACTTGGTGGTTTGCTACG & 197 \\
IL- 4 & GTGCAGCTTATCGATGAATCC & AGCCATATCCACGGATGCGAC & 287 \\
\hline
\end{tabular}




\section{Detection of Cytokine Production}

The serum from infected mice was separated by centrifugation at $1,000 \times g$ at $4^{\circ} \mathrm{C}$ for $10 \mathrm{~min}$ and then stored at $-80^{\circ} \mathrm{C}$ until analysis. Serum IFN- $\gamma, \operatorname{IgG}$, IgG1, and IgG2a were detected by mouse IFN- $\gamma, \operatorname{IgG}, \operatorname{IgG} 1$, and IgG2a ELISA kits, respectively, according to the manufacturer's (Multi Sciences [Lianke], China) instructions.

\section{Statistical Analysis}

Statistical analysis was performed using SPSS version 23 software. All results are presented as standard deviations (SD) and were repeated at least three times. Statistical significance was performed using unpaired, twotailed Student's $t$-test or two-way analysis of variance (ANOVA) followed by either Bonferroni's or Sidak's multiple-comparison test. Probability $(p)<0.05$ was considered statistically significant. $p \leq 0.01$ are denoted by ${ }^{* *}$; $0.01<p<0.05$ are denoted by ${ }^{*}$.

\section{Ethics Statement}

All animal experiments were conducted in accordance with the "Guidelines on Ethical Treatment of Experimental Animals" (2006) No. 398 from the Ministry of Science and Technology, China. The sampling procedures used in the study received prior approval from the Experimental Animal Management Committee of Northwest A\&F University with the approval license number 2018ZX08018023.

\section{Results}

\section{Analysis of Spleen Morphology}

The spleen is the main target organ for colonization and is also the crucial marker of residual bacterial virulence in Brucella-infected mice. To determine the effect of the Omp16 protein on bacterial virulence in vivo, six-toeight-week old female BALB/c mice were injected with approximately $1 \times 10^{7} \mathrm{CFU}$ of B. suis S2 or the $\Delta \mathrm{Omp} 16$ strain. The spleens of mice were enlarged significantly in both wild-type strain-infected mice and $\Delta$ Omp16infected mice compared to uninfected mice at one week post-infection, but splenomegaly was not significantly different between mice infected with either wild-type strain or $\Delta \mathrm{Omp} 16$ (Fig. 1A). The spleens of mice still were enlarged significantly following either wild-type strain or $\Delta \mathrm{Omp} 16$ infection compared to uninfected mice after two weeks, but splenomegaly at this time point was reduced in $\Delta$ Omp16-infected mice compared to mice infected with the parental strain (Fig. 1A). The spleens of both wild-type strain- and $\Delta$ Omp16-infected mice were fully restored at four weeks post-infection (Fig. 1A). Consistently, spleen weights were increased in both wild-type strain- and $\Delta$ Omp16-infected mice at one and two weeks post-infection, but were fully restored four weeks after infection (Fig. 1B). However, spleen weight was reduced following $\Delta$ Omp16 infection compared to wild-type strain infection at the two-week post-infection time point (Fig. 1B). These results demonstrate that Omp16 is involved in Brucella virulence in vivo.

\section{Omp16 Deficiency Impairs Brucella Survival in the Mouse Spleen}

Virulent strains of Brucella, including B. abortus strain 2308 and B. melitensis strain 16M, persist in the mouse spleen and cause long-term infection. However, live attenuated Brucella strains, such as B. abortus strains RB51

\section{A}
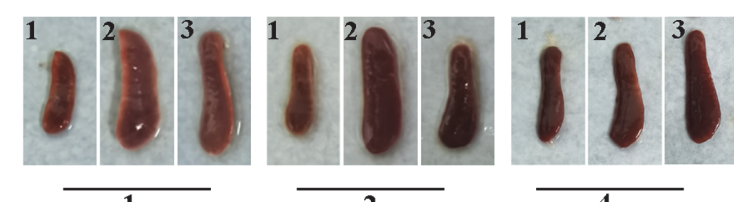

Weeks post infection

B

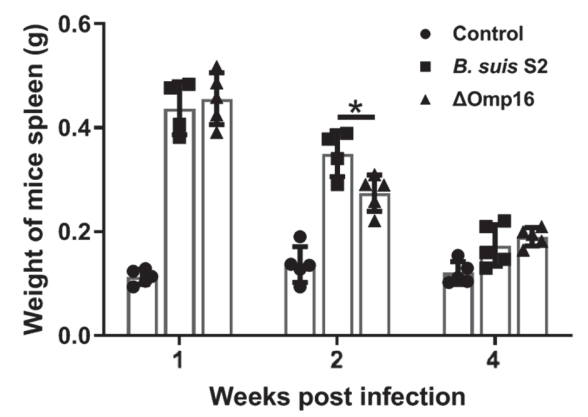

Fig. 1. Splenomegaly and spleen weights of mice infected with $B$. suis 2 wild-type or the $\Delta$ Omp16 strain. (A) Splenomegaly was observed at one, two and four weeks post-infection. 1, spleen of PBS-injected mice; 2, spleen of wild-type strain-infected mice; 3 , spleen of $\Delta$ Omp16-infected mice. The image shown is representative of at least five independent experiments. (B) Spleen weights were measured at one, two and four weeks post-infection. The results are expressed as the means \pm standard deviations $(\mathrm{SD})$. 
A

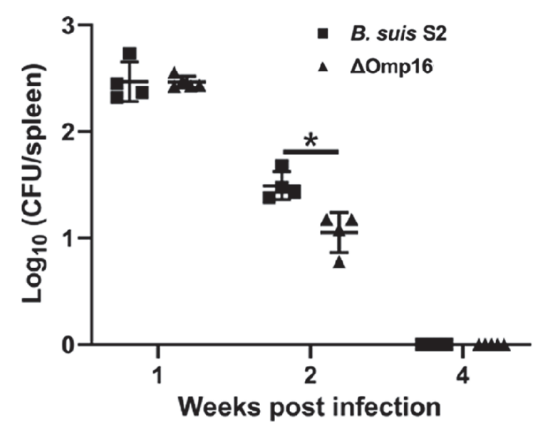

B control

B. suis $\mathrm{S2}$ $\Delta$ Omp16

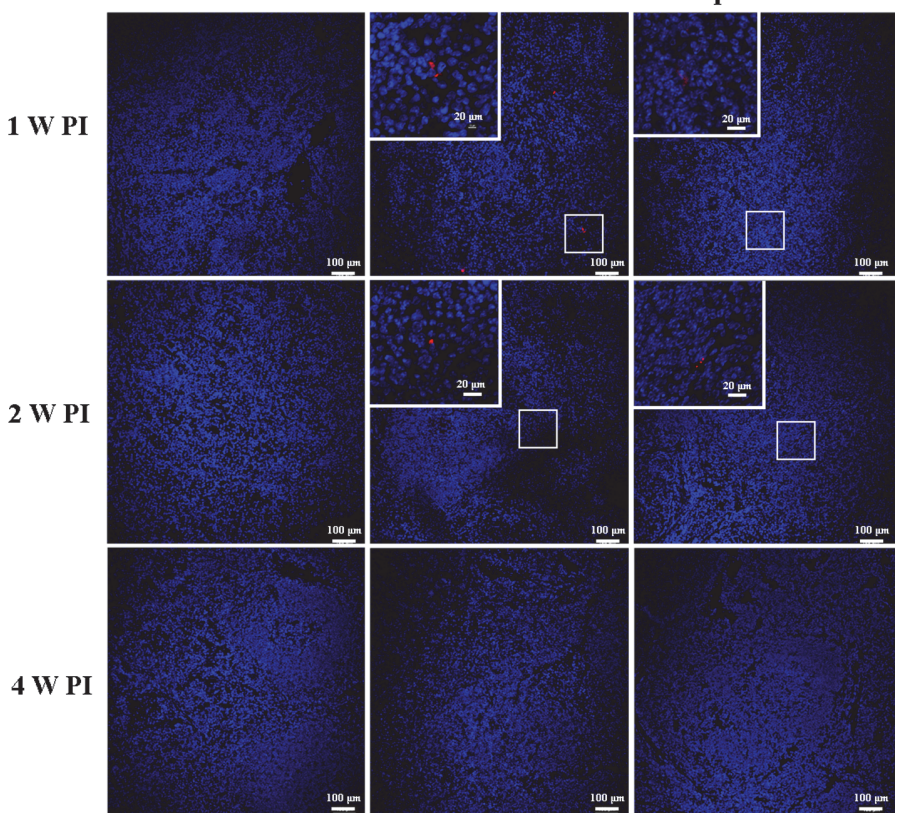

Fig. 2. Bacterial survival in the spleens of infected mice. (A) The number of bacteria was measured via homogenate at one, two and four weeks post-infection. The results are expressed as the means \pm SD. (B) Confocal microscopy analysis for bacterial survival in spleen of infected mice at one, two and four weeks post-infection. Red indicates wild-type strain or $\Delta$ Omp16 whereas the cell nucleus stains blue. The image shown is representative of at least three independent experiments.

and S19, B. melitensis strain Rev1, and B. suis strain S2, are eradicated quickly [11, 31, 32]. Based on spleen morphology, we hypothesized that Omp16 might affect Brucella survival in the mouse spleen. To this end, the survival of the $\Delta$ Omp16 strain in the spleen was examined by determining the CFU number. The numbers of bacteria decreased from one week post-infection to four weeks post-infection in the spleen of both wild-type strain- and $\Delta$ Omp16-infected mice (Fig. 2A). The bacterial loads were not significantly different between the wild-type strain and $\Delta$ Omp16 strains one week after infection, but the $\Delta$ Omp16 strain survived less well two weeks post-infection compared to the parental strain (Fig. 2A). The bacteria were completely eradicated from the spleen in both cases four weeks after infection (Fig. 2A). These results were confirmed by confocal microscopy analysis (Fig. 2B). The results overall indicate that Omp16 is required for efficient survival of B. suis in the mouse spleen.

\section{Pathological Analysis of Mice Immunized with $\Delta$ Omp16}

In view of the preceding characterization of the $\Delta$ Omp16 strain in the spleen, histopathologic examination was performed to investigate further the effect of the Omp16<Omp16> deletion on infectivity in mice. To this end, spleen tissues from mice infected either with the $S 2$ strain or $\Delta \mathrm{Omp} 16$ strain were collected at intervals of one, two, and four weeks post-infection and were fixed, mounted, and subjected to H\&E staining to assess tissue sample structure. No significant pathological changes were observed in uninfected spleens at the three time points (Fig. 3). However, spleens infected with the parental strain exhibited a significant increase in the white-to-red pulp ratio due to white pulp expansion at one and two weeks post-infection, although this effect was fully restored four weeks after infection (Fig. 3). In parallel, macrophage numbers increased in the red pulp of spleens of wildtype strain-infected mice after one week compared to uninfected mouse spleens (Fig. 3). In contrast, infection with the $\Delta$ Omp16 strain reduced the pathological characteristics of the spleen compared to infection with wild- 


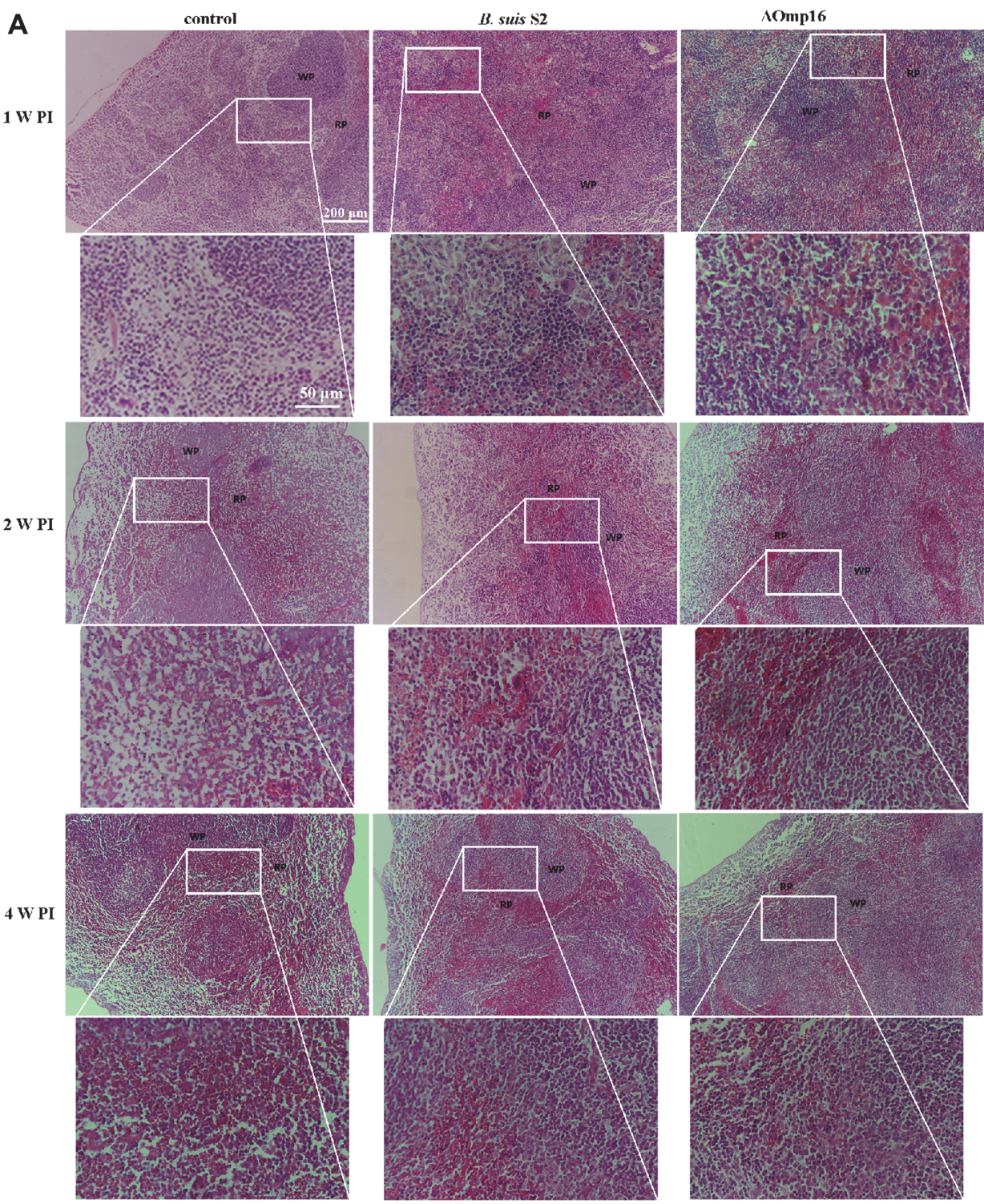

B

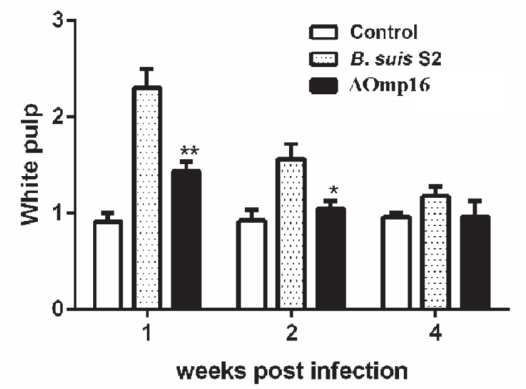

Fig. 3. Histopathology of the spleen at one, two and four weeks post-infection with wild-type strain or $\Delta$ Omp16. Spleen tissues from infected mice were collected at one, two and four weeks post-infection. After fixation, the tissues were embedded in paraffin blocks which were then sectioned into $5 \mu \mathrm{m}$ slices using a microtome. The sections were transferred to glass slides and allowed to adhere prior to further processing. The pathological features were assessed via H\&E staining. Arrows represent macrophages. WP, White pulp; RP, red pulp.

type strain with a modest increase in the white-to-red pulp ratio and slight white pulp expansion at one week postinfection (Fig. 3). In parallel, macrophage numbers increased in the red pulp of $\Delta$ Omp16-infected spleens at one 

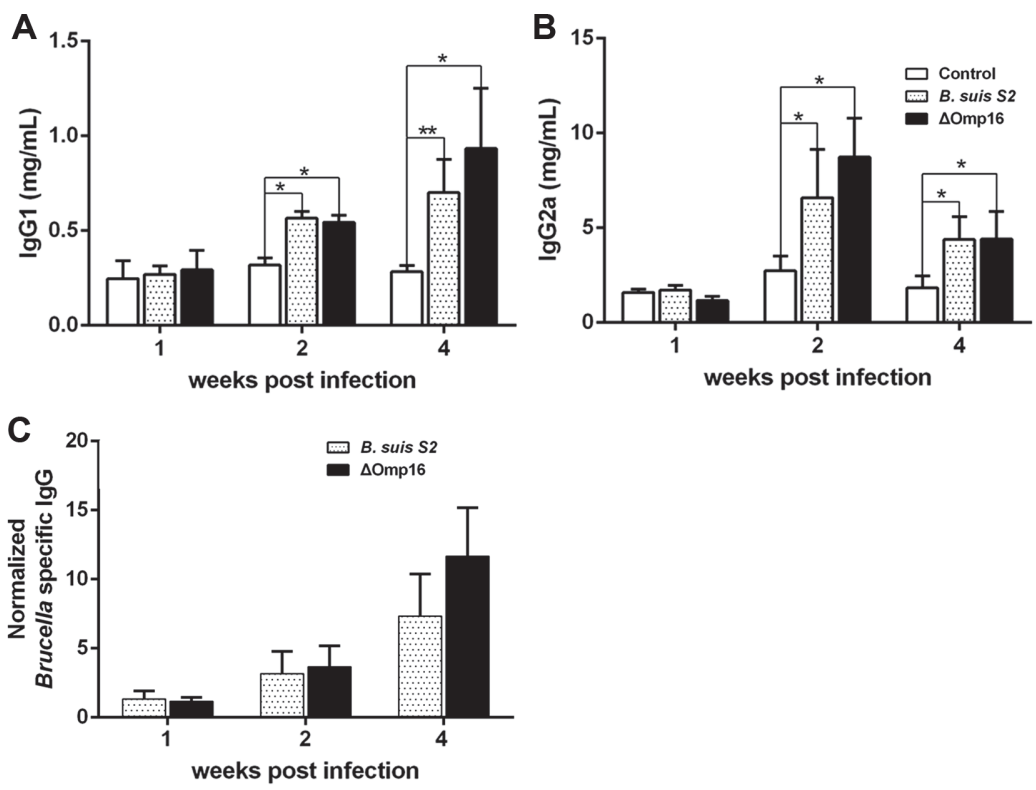

Fig. 4. Humoral immune response elicited in mice infected with wild-type strain or $\Delta$ Omp16. The serum from infected mice was collected and separated by centrifugation at $1,000 \times g$ at $4^{\circ} \mathrm{C}$ for $10 \mathrm{~min}$. IgG1 (A) and IgG2a (B) were measured at one, two and four weeks post-infection with ELISA kits. The results are expressed as the means \pm SD from three independent experiments.

week post-infection (Fig. 3). The data show that the pathological characteristics elicited by wild-type strain were ameliorated in spleens infected with the $\Delta$ Omp16 strain.

\section{Humoral and Cellular Immune Responses Elicited by Vaccination}

To further assess the effect of Omp16 on the humoral and cellular immune responses, the spleens from infected mice were collected and IgG1 and IgG2 secretion was detected by ELISA and inflammatory cytokine levels were

A

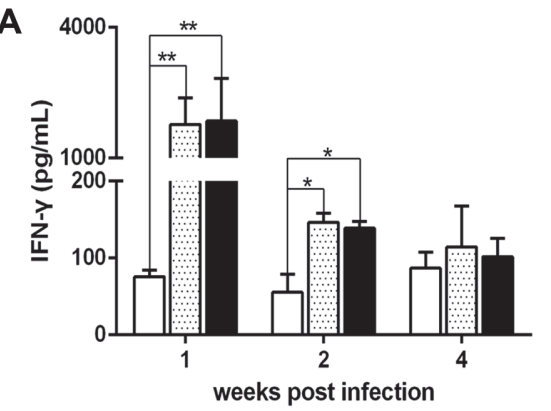

C

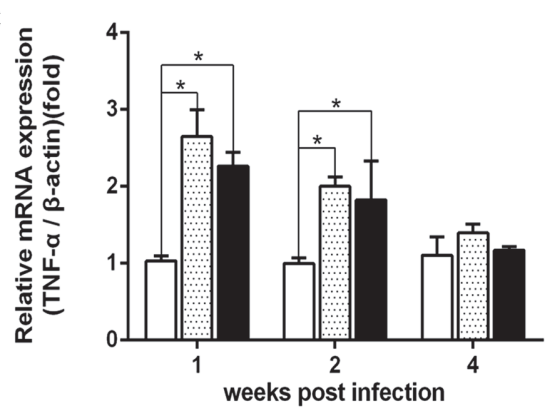

B

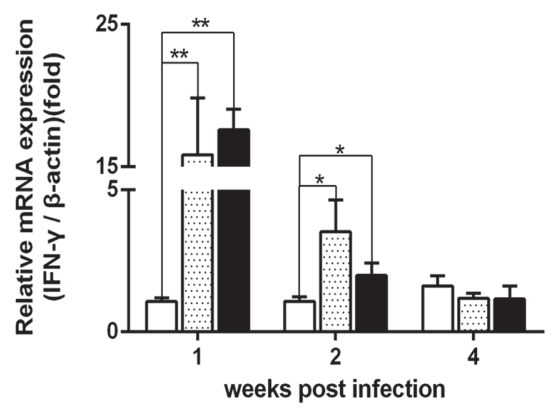

D

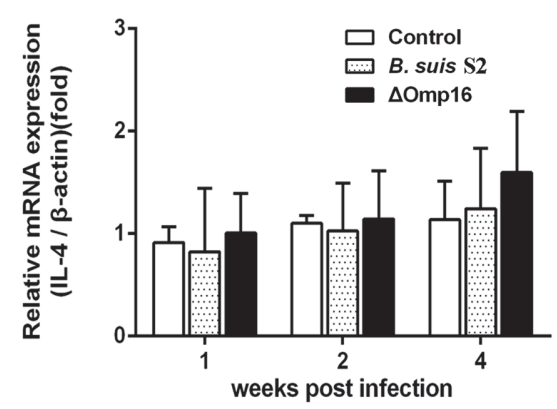

Fig. 5. Cytokine expression in serum and splenocytes in mice infected with wild-type strain or $\Delta$ Omp16. (A) The secretion of IFN- $\gamma$ in serum of mice was assayed with ELISA kits at one, two and four weeks post-infection. The results are expressed as the means \pm SD from three independent experiments. The mRNA levels of IFN- $\gamma$ (B), TNF- $\alpha$ (C) and IL-4 (D) were assayed by qRT-PCR at one, two and four weeks post-infection. The results are expressed as the means \pm SD from three independent experiments. 
assessed by qRT-PCR. The secretion of IgG1 and IgG2 from serum was increased in wild-type strain-infected mice at two and four weeks post-infection compared to uninfected mice (Fig. 4A). Similarly, the secretion of IgG1 and IgG2 from serum was increased in $\Delta$ Omp16-infected mice (Fig. 4A). There was no significant difference in IgG1 and IgG2 levels between wild-type strain- and $\Delta$ Omp16-infected mice at one, two or four weeks postinfection (Fig. 4A). These results demonstrate that the level of humoral immunity in $\Delta$ Omp 16 -infected mice was similar to that in mice infected with the parental strain. Moreover, secretion of IFN- $\gamma$ from serum was elevated to similar levels in both parental strain- and $\Delta$ Ompl6-infected mice after one and two weeks compared to uninfected mice (Fig. 5A). The expression of IFN- $\gamma$ mRNA in the spleen was consistent with the IFN- $\gamma$ levels that were detected in serum (Fig. 5B). In addition, the expression of TNF- $\alpha$ mRNA was increased in parental strain and $\Delta$ Omp16-infected spleens one and two weeks post-infection compared to uninfected mice (Fig. 5C), although there was no significant difference between the two infected samples (Fig. 5C). Finally, we observed that IL-4 expression did not change significantly in spleens infected with either the parental or deletion strain compared to control spleens. Overall, the results indicate that Omp16 did not affect IFN- $\gamma$, TNF- $\alpha$ and IL-4 secretion during infection by B. suis.

\section{Discussion}

Brucellosis, caused by Brucella spp., is a worldwide zoonotic and contagious disease that causes abortion and infertility in animals and chronic debilitating disease in humans that results in serious morbidity, worldwide economic loss, and poverty [7-9]. The available live attenuated vaccines still have some disadvantages in animals, such as serodiagnostic interference and residual pathogenicity [11-13]. In contrast, no patient-friendly therapeutic methods or approved vaccines are reported for humans [9]. Omps, including Omp10, Omp19, Omp25 and Omp31, are involved in outer membrane integrity, virulence and evasion of the host immune system in Brucella [24, 25, 28, 33, 34]. Exploration of the function of Omps has been key to understand the pathogenic mechanisms of Brucella and the ability of this bacterium to evade the immune system. Omp16, a homolog of peptidoglycan-associated lipoproteins (Pals), is a key factor for Brucella survival [28]. A Brucella strain deleted of the gene for Omp16 has not been generated in any species. In a previous study, we acquired the $\Delta \mathrm{Omp} 16$ strain in which omp16 expression is controlled [29]. Here, we utilized this strain to explore the effect of Omp16 on bacterial virulence and the Brucella-mediated immune response in mice. Our results showed that Omp16 is required for Brucella survival in the spleen and that the humoral and cellular immune responses mediated by $\Delta$ Omp 16 are similar to those of the wild-type strain.

The mouse is an important model for persistent Brucella infection [7]. Splenomegaly, spleen weight, and survival of bacteria in the spleen are key indicators to assess Brucella virulence. Several Brucella Omps have been confirmed to be involved in bacterial virulence. For example, the intracellular survival of an Omp10 and Omp19 deletion strain is decreased in the spleen, indicating that this strain is attenuated in vivo $[23,24,35]$. Moreover, Omp25 is an important virulence factor in Brucella and a strain deleted of omp25 is attenuated both in vitro and in vivo [36]. Furthermore, Omp31 plays an important role in Brucella virulence [37, 38]. However, several other Omps in Brucella, including SP41 and BepC, are not involved in virulence [28]. In a previous study, we found that Brucella cells lacking Omp16 presented defects in growth, outer membrane integrity and intracellular survival [29]. Further studies are required to explore the virulence of $\Delta$ Omp16 in mice. Using the BALB/c mouse model, we showed that splenomegaly and spleen weight were reduced in $\Delta$ Omp16-infected mice and that the bacteria in these mice were cleared quickly compared to parental strain-infected mice. These results are similar to the characteristics of other Omp deletion strains described above. As another key indicator, spleens from $\Delta$ Omp16infected mice exhibited reduced pathological characteristics with a modest increase in the white-to-red pulp ratio and slight white pulp expansion one week post-infection compared to spleens of mice infected with the parental strain. These observations indicate that Omp 16 is required to maintain B. suis virulence in mice and that $\Delta \mathrm{Omp} 16$ is attenuated in mice compared to the wild-type strain. The Brucella outer membrane is resistant to bactericidal cationic peptide and bactericidal polycations like polymyxin B [39]. Brucella LPS, Omp31 and Omp16 mutant strain were more susceptible to polymyxin B in vitro [29,37, 40]. In addition, intracellular bacteria have developed the capability to adapt to intracellular environments to survive in host cells, including oxidative burst [26]. The survival rate of Brucella Omp16 mutant was decreased under oxidative stress [29]. The reduced virulence of the $\Delta$ Omp16 in a mouse model of infection may be due to changes in the biological characteristics of bacteria. These findings corroborate our previous results that the intracellular survival rate of $\Delta \mathrm{Omp} 16$ is decreased compared to wild-type in RAW264.7 macrophages [29].

The humoral response is required to resist intracellular pathogenic bacteria and contributes to the control of bacterial infection [41, 42]. The secretion of IgG2a antibody is associated with cytokines secreted by Th1-type cells, whereas the production of IgG1 antibody is associated with cytokine secretion by Th2-type cells [43]. Wildtype strain and $\Delta$ Omp16 induced higher production of IgG1 and IgG2a in infected vs. uninfected mice. However, the production of IgG1 and IgG2a was not significantly different in $\Delta$ Omp16-infected mice compared to wildtype strain-infected mice which indicates that Omp16 is not implicated in the humoral response to Brucella infection. During Brucella infection in the murine model, the host immune response resembles the Th1 type with the secretion of IFN- $\gamma$ and TNF- $\alpha$ by infected macrophages $[44,45]$. As a key cytokine, IFN- $\gamma$ plays an important role in resisting intracellular pathogenic bacteria, including Brucella [46]. IFN- $\gamma^{-/-}$mice are unable to control infections, resulting in the death of infected mice at six weeks post-infection [47]. Unlipidated Omp16 (obtained from the bacterial cytoplasm) is able to induce higher protection levels in vivo than lipidated Omp16 (obtained from bacterial membranes), and unlipidated Omp16 mediates IFN- $\gamma$ production to eradicate Brucella [27]. However, we did not observe that the secretion of IFN- $\gamma$ was significantly different in $\Delta$ Ompl6-infected mice 
compared to mice infected with parental strain. This may be due to poor induction of IFN- $\gamma$ secretion by lipidated Omp16. Furthermore, TNF- $\alpha$ contributes to the control of intracellular pathogenic bacteria. However, neither the secretion of TNF- $\alpha$ nor the production of IL- 4 was different in $\Delta$ Omp16-infected mice compared to wild-type strain-infected mice. Overall, the results show that the levels of humoral and cellular immunity in wild-type strain- and $\Delta$ Omp16-infected mice were similar.

In conclusion, the current study reveals that murine splenomegaly, bacterial load, and histopathological changes in spleen were significantly decreased in a strain deleted of the gene for Omp16 compared to wild-type strain. In addition, the levels of humoral and cellular immunity were indistinguishable in wild-type strain- and $\Delta$ Omp16-infected mice. Overall, these findings reveal that Omp16 is required for Brucella survival in mice, but that Omp16 is not involved in Brucella-induced humoral and cellular immunity. Future studies will focus further on the key role of Omp16 in infection by B. suis.

\section{Acknowledgments}

This research was funded by the National Key R\&D Program of China (2018YFD0500900); the National Natural Science Foundation of China (31672584, 31702310).

\section{Conflict of Interest}

The authors have no financial conflicts of interest to declare.

\section{References}

1. Franc KA, Krecek RC, Hasler BN, Arenas-Gamboa AM. 2018. Brucellosis remains a neglected disease in the developing world: a call for interdisciplinary action. BMC Public Health 18: 125.

2. Bialer MG, Sycz G, Gonzalez FM, Ferrero MC, Baldi PC, Zorreguieta A. 2020. Adhesins of Brucella: Their roles in the interaction with the host. Pathogens 9: 942

3. Al Dahouk S, Scholz HC, Tomaso H, Bahn P, Gollner C, Karges W, et al. 2010. Differential phenotyping of Brucella species using a newly developed semi-automated metabolic system. BMC Microbiol. 10: 269.

4. Foster G, Osterman BS, Godfroid J, Jacques I, Cloeckaert A. 2007. Brucella ceti sp. nov. and Brucella pinnipedialis sp. nov. for Brucella strains with cetaceans and seals as their preferred hosts. Int. J. Syst. Evol. Microbiol. 57: 2688-2693.

5. Scholz HC, Hubalek Z, Sedlacek I, Vergnaud G, Tomaso H, Al Dahouk S, et al. 2008. Brucella microti sp. nov., isolated from the common vole Microtus arvalis. Int. J. Syst. Evol. Microbiol. 58: 375-382.

6. Lucero NE, Corazza R, Almuzara MN, Reynes E, Escobar GI, Boeri E, et al. 2010. Human Brucella canis outbreak linked to infection in dogs. Epidemiol. Infect. 138: 280-285.

7. Byndloss MX, Tsolis RM. 2016. Brucella spp. virulence factors and immunity, pp. 111-127. In Lewin HA, Roberts RM (eds.), Annual Review of Animal Biosciences, Vol 4, Ed.

8. Elfaki MG, Alaidan AA, Al-Hokail AA. 2015. Host response to Brucella infection: review and future perspective. J. Infect. Dev. Ctries 9: 697-701.

9. de Figueiredo P, Ficht TA, Rice-Ficht A, Rossetti CA, Adams LG. 2015. Pathogenesis and immunobiology of brucellosis review of Brucella-host interactions. Am. J. Pathol. 185: 1505-1517.

10. Weber MM, Faris R. 2018. Subversion of the endocytic and secretory pathways by bacterial effector proteins. Front. Cell Dev. Biol. 6: 1 .

11. Lalsiamthara J, Lee JH. 2017. Development and trial of vaccines against Brucella. J. Vet. Sci. 18: 281-290.

12. Avila-Calderon ED, Lopez-Merino A, Sriranganathan N, Boyle S, Contreras-Rodriguez A. 2013. A history of the development of Brucella vaccines. Biomed Res. Int. 2013: 743509.

13. Dorneles EMS, Sriranganathan N, Lage AP. 2015. Recent advances in Brucella abortus vaccines. Vet. Res. $46: 76$.

14. Moreno E, Moriyon I. 2002. Brucella melitensis: a nasty bug with hidden credentials for virulence. Proc. Natl. Acad. Sci. USA 99: 1-3.

15. Seleem MN, Boyle SM, Sriranganathan N. 2008. Brucella: a pathogen without classic virulence genes. Vet. Microbiol. 129: 1-14.

16. Glowacka P, Zakowska D, Naylor K, Niemcewicz M, Bielawska-Drozd A. 2018. Brucella - virulence factors, pathogenesis and treatment. Pol. J. Microbiol. 67: 151-161.

17. de Jong MF, Starr T, Winter MG, den Hartigh AB, Child R, Knodler LA, et al. 2013. Sensing of bacterial type IV secretion via the unfolded protein response. $m$ Bio 4 : e00418-12.

18. Miller CN, Smith EP, Cundiff JA, Knodler LA, Blackburn JB, Lupashin V, et al. 2017. A brucella type IV effector targets the COG tethering complex to remodel host secretory traffic and promote intracellular replication. Cell Host Microbe 22: 317-329.

19. Bohin JP. 2000. Osmoregulated periplasmic glucans in Proteobacteria. FEMS Microbiol. Lett. 186: 11-19.

20. Caro-Hernandez P, Fernandez-Lago L, de Miguel M-J, Martin-Martin AI, Cloeckaert A, Grillo M-J, et al. 2007. Role of the Omp25/ Omp31 family in outer membrane properties and virulence of Brucella ovis. Infect. Immun. 75: 4050-4061.

21. Ibanez AE, Smaldini P, Coria LM, Delpino MV, Pacifico LG, Oliveira SC, et al. 2013. Unlipidated outer membrane protein Omp16 (U-Omp16) from Brucella spp. as nasal adjuvant induces a Th1 immune response and modulates the Th2 allergic response to cow's milk proteins. PLoS One 8: e69438.

22. Letesson JJ, Lestrate P, Delrue RM, Danese I, Bellefontaine F, Fretin D, et al. 2002. Fun stories about Brucella: the "furtive nasty bug". Vet. Microbiol. 90: 317-328.

23. Li TS, Huang ML, Wang Z, Guo F, Zhang H, Chen CF. 2017. Construction and characteristic analysis of Omp10 deletion mutant of Brucella abortus. Pak J. Zool. 49: 1809-1816.

24. Tibor A, Wansard V, Bielartz V, Delrue RM, Danese I, Michel P, et al. 2002. Effect of omp10 or omp19 deletion on Brucella abortus outer membrane properties and virulence in mice. Infect. Immun. 70: 5540-5546.

25. Pasquevich KA, Carabajal MV, Guaimas FF, Bruno L, Roset MS, Coria LM, et al. 2019. Omp19 enables Brucella abortus to evade the antimicrobial activity from host's proteolytic defense system. Front. Immunol. 10: 1436.

26. Wang YF, Chen ZL, Qiao F, Zhong ZJ, Xu J, Wang ZJ, et al. 2010. The type IV secretion system affects the expression of Omp25/ Omp31 and the outer membrane properties of Brucella melitensis. FEMS Microbiol. Lett. 303: 92-100.

27. Pasquevich KA, Garcia Samartino C, Coria LM, Estein SM, Zwerdling A, Ibanez AE, et al. 2010. The protein moiety of Brucella abortus outer membrane protein 16 is a new bacterial pathogen-associated molecular pattern that activates dendritic cells in vivo, induces a Th1 immune response, and is a promising self-adjuvanting vaccine against systemic and oral acquired brucellosis. J. Immunol. 184: 5200-5212. 
28. Sidhu-Munoz RS, Sancho P, Vizcaino N. 2016. Brucella ovis PA mutants for outer membrane proteins Omp10, Omp19, SP41, and BepC are not altered in their virulence and outer membrane properties. Vet. Microbiol. 186: 59-66.

29. Zhi FJ, Zhou D, Li JM, Tian LL, Zhang GD, Jin YP, et al. 2020. Omp16, a conserved peptidoglycan-associated lipoprotein, is involved in Brucella virulence in vitro. J. Microbiol. 58: 793-804.

30. Yang L, Ma T, Zhao L, Jiang H, Zhang J, Liu D, et al. 2021. Circadian regulation of apolipoprotein gene expression affects testosterone production in mouse testis. Theriogenology 174: 9-19.

31. Smither SJ, Perkins SD, Davies C, Stagg AJ, Nelson M, Atkins HS. 2009. Development and characterization of mouse models of infection with aerosolized Brucella melitensis and Brucella suis. Clin. Vaccine Immunol. 16: 779-783.

32. Zhu LQ, Feng Y, Zhang G, Jiang H, Zhang Z, Wang N, et al. 2016. Brucella suis strain 2 vaccine is safe and protective against heterologous Brucella spp. infections. Vaccine 34: 395-400.

33. Luo X, Zhang X, Wu X, Yang X, Han C, Wang Z, et al. 2017. Brucella downregulates tumor necrosis factor-alpha to promote intracellular survival via Omp25 regulation of different microRNAs in porcine and murine macrophages. Front. Immunol. 8: 2013.

34. Tabynov K, Sansyzbay A, Kydyrbayev Z, Yespembetov B, Ryskeldinova S, Zinina N, et al. 2014. Influenza viral vectors expressing the Brucella OMP16 or L7/L12 proteins as vaccines against B. abortus infection. Virol. J. 11: 69.

35. Wang YL, Ma ZC, Zhang H, Yi JH, Wang Y, Li TS, et al. 2020. The Deletion of Omp19 gene of Brucella abortus 2308 reduces its survival in mouse macrophage and in mice. Kafkas Univ. Vet. Fak. 26: 749-755.

36. Edmonds MD, Cloeckaert A, Elzer PH. 2002. Brucella species lacking the major outer membrane protein Omp25 are attenuated in mice and protect against Brucella melitensis and Brucella ovis. Vet. Microbiol. 88: 205-221.

37. Verdiguel-Fernandez L, Oropeza-Navarro R, Basurto-Alcantara F, Castaneda-Ramirez A, Verdugo-Rodriguez A. 2017. Omp31 plays an important role on outer membrane properties and intracellular survival of Brucella melitensis in murine macrophages and HeLa cells. Arch. Microbiol. 199: 971-978.

38. Verdiguel-Fernandez L, Oropeza-Navarro R, Ortiz A, Robles-Pesina MG, Ramirez-Lezama J, Castaneda-Ramirez A, et al. 2020. Brucella melitensis omp31 mutant is attenuated and confers protection against virulent Brucella melitensis challenge in $\mathrm{BALB} / \mathrm{c}$ mice. J. Microbiol. Biotechnol. 30: 497-504.

39. Sola-Landa A, Pizarro-Cerda J, Grillo MJ, Moreno E, Moriyon I, Blasco JM, et al. 1998. A two-component regulatory system playing a critical role in plant pathogens and endosymbionts is present in Brucella abortus and controls cell invasion and virulence. Mol. Microbiol. 29: 125-138.

40. Feng Y, Peng XW, Jiang H, Peng Y, Zhu LQ, Ding JB. 2017. Rough Brucella strain RM57 is attenuated and confers protection against Brucella melitensis. Microb. Pathog. 107: 270-275.

41. Vitry MA, Mambres DH, De Trez C, Akira S, Ryffel B, Letesson JJ, et al. 2014. Humoral immunity and CD4(+) Th1 cells are both necessary for a fully protective immune response upon secondary infection with Brucella melitensis. I. Immunol. 192: 3740-3752.

42. Casadevall A, Pirofski LA. 2006. A reappraisal of humoral immunity based on mechanisms of antibody-mediated protection against intracellular pathogens. Adv. Immunol. 91: 1-44.

43. Vidarsson G, Dekkers G, Rispens T. 2014. IgG subclasses and allotypes: from structure to effector functions. Front. Immunol. 5: 520.

44. Rolan HG, Tsolis RA. 2008. Inactivation of the type IV secretion system reduces the Th1 polarization of the immune response to Brucella abortus infection. Infect. Immun. 76: 3207-3213.

45. Zhan YF, Liu ZQ, Cheers C. 1996. Tumor necrosis factor alpha and interleukin-12 contribute to resistance to the intracellular bacterium Brucella abortus by different mechanisms. Infect. Immun. 64: 2782-2786.

46. Rubach MP, Halliday JEB, Cleaveland S, Crump JA. 2013. Brucellosis in low-income and middle-income countries. Curr. Opin. Infect. Dis. 26: 404-412.

47. Murphy EA, Sathiyaseelan J, Parent MA, Zou BX, Baldwin CL. 2001. Interferon-gamma is crucial for surviving a Brucella abortus infection in both resistant C57BL/6 and susceptible BALB/c mice. Immunology 103: 511-518. 